\title{
THE INCLUSION OF AN ELEVATED PEAK C-REACTIVE PROTEIN IN THE CASE DEFINITION OF LATE NEONATAL SEPSIS IDENTIFIES A SIGNIFICANT NUMBER OF CULTURE NEGATIVE SEPTIC EPISODES
}

\author{
B. Schoonakker ${ }^{1}$, K. Swamy ${ }^{1}$, H. Budge ${ }^{2}$ \\ ${ }^{l}$ Neonatal Intensive Care Unit, Nottingham University NHS Trust, ${ }^{2}$ Early Life Nutrition Research Unit, \\ Academic Child Health, University of Nottingham, Nottingham, UK
}

Background and aims: For the purposes of infection surveillance, late-neonatal septic episodes are usually defined as a positive culture from a normally sterile site occurring 72 hours after birth for which a clinician has prescribed five days of antibiotics ${ }^{1}$. Culture negative septic episodes are, however, not recorded and their contribution to the sepsis burden is unknown.

Methods: Over a six month period, all episodes of possible late sepsis on a Level 3 NICU were prospectively identified and classified according to peak C-reactive protein levels, blood and other culture results. Repeat episodes were identified and excluded.

Results: 247 unique episodes were identified. Of these, 18 episodes had a positive culture and elevated CRP (Group B positive Streptococcus (1), Klebsiella pneumonia (2), coagulase negative Staphylococci (15)). In 51 episodes, there was an elevated CRP but negative cultures. Of these, 36 were considered to be highly clinically suspicious of sepsis and 11, were considered to result from non-infected causes (e.g. immunisation). 15 of the 22 with positive blood cultures without rise in CRP were treated by the attending clinician with at least 5 days of antibiotics (all coagulase negative Staphylococci).

\begin{tabular}{|c|c|c|}
\hline & Blood Culture Positive & Blood Culture Negative \\
\hline \hline CRP $\geq \mathbf{1 0}$ & 18 & 51 \\
\hline CRP $<\mathbf{1 0}$ & 22 & 156 \\
\hline
\end{tabular}

Conclusions: A rise in CRP during a septic episode allowed the identification of additional clinically significant culture negative septic episodes.

(1) Vergnano et al. Arch Dis Child 2010 96: F9-F14 